\title{
Homogeneous Gödel-type solutions in hybrid metric-Palatini gravity
}

\author{
J. Santos ${ }^{1, a}$, M. J. Rebouças ${ }^{2, b}$, A. F. F. Teixeira ${ }^{2}$ \\ ${ }^{1}$ Departamento de Física Teórica e Experimental, Universidade Federal do Rio Grande do Norte, Natal, RN 59072-970, Brazil \\ ${ }^{2}$ Centro Brasileiro de Pesquisas Físicas, Rua Dr. Xavier Sigaud 150, Rio de Janeiro, RJ 22290-180, Brazil
}

Received: 10 May 2018 / Accepted: 25 June 2018 / Published online: 10 July 2018

(C) The Author(s) 2018

\begin{abstract}
The hybrid metric-Palatini $f(\mathscr{R})$ gravity is a recently devised approach to modified gravity in which it is added to the metric Ricci scalar $R$, in the Einstein-Hilbert Lagrangian, a function $f(\mathscr{R})$ of Palatini curvature scalar $\mathscr{R}$, which is constructed from an independent connection. These hybrid metric-Palatini gravity theories provide an alternative way to explain the current accelerating expansion without a dark energy matter component. If gravitation is to be described by a hybrid metric-Palatini $f(\mathscr{R})$ gravity theory there are a number of issues that ought to be examined in its context, including the question as to whether its equations allow homogeneous Gödel-type solutions, which necessarily leads to violation of causality. Here, to look further into the potentialities and difficulties of $f(\mathscr{R})$ theories, we examine whether they admit Gödel-type solutions for physically well-motivated matter source. We first show that under certain conditions on the matter sources the problem of finding out space-time homogeneous (ST-homogeneous) solutions in $f(\mathscr{R})$ theories reduces to the problem of determining solutions of Einstein's field equations with a cosmological constant. Employing this far-reaching result, we determine a general ST-homogeneous Gödel-type solution whose matter source is a combination of a scalar with an electromagnetic fields plus a perfect fluid. This general Gödel-type solution contains special solutions in which the essential parameter $m^{2}$ can be $m^{2}>0$ hyperbolic family, $m=0$ linear class, and $m^{2}<0$ trigonometric family, covering thus all classes of homogeneous Gödel-type spacetimes. This general solution also contains all previously known solutions as special cases. The bare existence of these Gödel-type solutions makes apparent that hybrid metric-Palatini $f(\mathscr{R})$ gravity does not remedy causal anomaly in the form of closed timelike curves that are permitted in general relativity.
\end{abstract}

\footnotetext{
a e-mail: janilo.santos@gmail.com

be-mail: reboucas@cbpf.br
}

\section{Introduction}

A number of cosmological observations coming from different sources, including the supernovae type Ia (SNe Ia) [1-3], the cosmic microwave background radiation (CMBR) $[4,5]$ and baryon acoustic oscillation (BAO) surveys [6-10], indicate that the Universe is presently expanding with an accelerating rate. The frameworks proposed to account for this observed accelerated expansion can be roughly grouped into two families. In the first, the underlying theory, general relativity (GR), is kept unchanged, and the so-called dark energy component is invoked. In this context, the simplest way to account for the accelerating expansion of the Universe is through the introduction of a cosmological constant, $\Lambda$, into Einstein's field equations. This is completely consistent with the available observational data, but it faces difficulties such as the order of magnitude of the cosmological constant and its microphysical origin. In the second family, modifications of Einstein's field equations are assumed as an alternative for explaining the accelerated expansion. This latter group includes, for example, generalized theories of gravity based upon modifications of the Einstein-Hilbert action by taking nonlinear functions, $f(R)$, of the Ricci scalar $R$ or other curvature invariants (for reviews see Refs. [11-16]).

In dealing with $f(R)$ gravity theories two different variational approaches, which give rise to different dynamics, have often been considered in the literature [11-16]. In the so-called metric formalism the connection is assumed to be Levi-Civita, therefore defined by the metric. In the Palatini formalism the metric and the connection are treated as independent fields, and it is assumed that the matter fields do not couple with the independent connections. Although these approaches have been invoked as possible ways to satisfactorily deal with the observed accelerated expansion of the Universe, it has been pointed out that $f(R)$ gravity theories can face relevant difficulties, including the evolution of cos- 
mological perturbations and local gravity constraints (see, for example, Refs. [14, 17-22]).

These undesirable features have motivated a recent approach to modified $f(R)$ theories of gravity, which can be employed as a possible way to handle the observed latetime cosmic acceleration, and also circumvent some difficulties that arise in the framework of $f(R)$ theories in both formalisms. The hybrid metric-Palatini $f(\mathscr{R})$ gravity is a recently devised approach to such modified theories, in which it is added to the ordinary Ricci scalar $R$, in the EinsteinHilbert Lagrangian, a function, $f(\mathscr{R})$, of Palatini curvature scalar $\mathscr{R}$, which is constructed from the independent connection $\Gamma_{\mu \nu}^{\rho}$ [23]. These hybrid metric-Palatini gravity theories appears to suitably unify the description of the late-time cosmic acceleration with the local solar system constraints $[23,24]$. Some of the astrophysical and cosmological implications of hybrid metric-Palatini gravity have been examined in a number of papers [25-29]. Wormwhole solutions, Einstein static universe, linear perturbations, the Cauchy problem, dynamical system analysis and a brane model have been discussed, respectively, in the references [30-35]. Other important matters such as Noether symmetries [36] and the thermodynamic behavior [37] have also been recently considered. For an introduction to hybrid metric-Palatini $f(\mathscr{R})$ gravity and a detailed list of related references, we refer the reader to the recent review article [28].

In general relativity (GR) the space-times have locally the same causal structure of the flat space-time of special relativity since the space-times of GR are locally Minkowskian. On nonlocal scale, however, significant differences may arise since the general relativity field equations do no provide nonlocal constraints on the underlying space-times. Indeed, it has long been known that there are solutions to Einstein's field equations that present nonlocal causal anomalies in the form of closed time-like curves (see, for example, Refs. [38-43].

The renowned model found by Gödel [44] is the best known example of a solution to the Einstein's equations, with a physically well-motivated source, that makes it apparent that GR permits solutions with closed timelike world lines, regardless of its local Lorentzian character that ensures locally an inherited regular chronology and therefore the local validation of the causality principle. The Gödel model is a solution of Einstein's equations with cosmological constant $\Lambda$ for dust of density $\rho$, but it can also be viewed as perfect-fluid solution with equation of state $p=\rho$ with no cosmological constant term. Owing to its unexpected properties, Gödel's solution has a recognizable importance and has motivated a considerable number of investigations on rotating Gödel-type models as well as on causal anomalies in the context of general relativity (see, e.g. Refs. [38-54] and other gravity theories [55-79]. In two recent papers, we have also examined Gödel-type models and the violation of causality problem for $f(R)$ gravity in both the metric and
Palatini variational approaches [71,72], extending therefore the results of Refs. [80,81].

If gravitation is to be described by hybrid metric-Palatini $f(\mathscr{R})$ gravity theory there are a number of issues that ought to be reexamined in its context, including its consistence with the recent detection of gravitational wave [82-84] and the question as to whether these gravity theories allow Gödeltype solutions, which necessarily lead to closed timelike curves, or would remedy this causal pathology by ruling out this type of solutions, which are permitted in general relativity.

In this article, to proceed further with the investigations on the potentialities, difficulties and limitations of $f(\mathscr{R})$, we undertake this question by examining whether the $f(\mathscr{R})$ gravity theories admit homogeneous Gödel-type solutions for a combination of physically well-motivated matter sources. To this end, we first examine the general problem of finding out ST-homogeneous solutions in hybrid metricPalatini $f(\mathscr{R})$ gravity for matter sources with constant trace $T$ (scalar) of the energy-momentum tensor, and show that it reduces to to the problem of determining ST-homogeneous solutions of Einstein's field equations with a cosmological constant determined by $f(\mathscr{R})$ and its first derivative $f^{\prime}(\mathscr{R})$. Employing this far-reaching result, we determine a general ST-homogeneous Gödel-type whose matter source is a combination of a scalar with an electromagnetic fields plus a perfect fluid. This general Gödel-type solution contains special solutions in which the essential parameter $m^{2}$ defines any one of the possible classified families homogeneous Gödeltype solutions, namely $m^{2}>0$ hyperbolic family, $m=0$ linear class, and $m^{2}<0$ trigonometric family. This general homogeneous Gödel-type solution also contains previously known solutions as special cases. There emerges from one of the particular solution of the hyperbolic family that every perfect-fluid Gödel-type solution of any $f(\mathscr{R})$ gravity with density $\rho$ and pressure $p$ and satisfying the weak energy conditions $\rho>0$ and $\rho+p \geq 0$ is necessarily isometric to the Gödel geometry. ${ }^{1}$

The bare existence of these noncausal Gödel-type solutions makes apparent that hybrid metric-Palatini $f(\mathscr{R})$ gravity does not remedy causal anomaly in the form of closed timelike curves that are permitted in general relativity.

The structure of the paper is as follows. In Sect. 2 we give a brief account of the hybrid metric-Palatini $f(\mathscr{R})$ gravity theories. In Sect. 3 we present the basic properties of homogenous Gödel-type geometries. This includes the conditions for space-time homogeneity, a classification of ST-homogeneous Gödel-type geometries and a study of the existence of closed time-like curves in all ST-homogeneous

\footnotetext{
1 This extends to the context of $f(\mathscr{R})$ gravity a theorem which states that every perfect-fluid Gödel-type solution of Einstein's equations is necessarily isometric to the Gödel spacetime [85].
} 
Gödel-type metrics. In Sect. 4 we first examine the problem of finding out ST-homogeneous solutions in $f(\mathscr{R})$ gravity whose trace $T$ of the energy-momentum tensor of the matter source is constant, and show that in such cases the problem reduces to that of finding out solutions of Einstein's field equations with a cosmological constant. We then show that the hybrid metric-Palatini $f(\mathscr{R})$ gravity theories admit SThomogeneous Gödel-type solutions for a general combination of physically well-motivated matter contents.

\section{Hybrid metric-Palatini gravity}

The action that defines a hybrid metric-Palatini gravity is given by

$S=\frac{1}{2 \kappa^{2}} \int d^{4} x \sqrt{-g}\left[R+f(\mathscr{R})+\mathscr{L}_{m}\right]$,

where $\kappa^{2}=8 \pi G, g$ is the determinant of the metric tensor $g_{\mu \nu}, R$ is the Ricci scalar associated to the Levi-Civita connection of the metric $g_{\mu \nu}, \mathscr{L}_{m}$ is the Lagragian density for the matter fields, and the extra term $f(\mathscr{R})$ is a function of Palatini curvature scalar $\mathscr{R}$, which depends on the metric and on an independent connection $\Gamma_{\mu \nu}^{\rho}$ through

$\mathscr{R} \equiv g^{\mu v} \mathscr{R}_{\mu v}=g^{\mu v}\left(\partial_{\rho} \Gamma_{\mu \nu}^{\rho}-\partial_{\nu} \Gamma_{\mu \rho}^{\rho}+\Gamma_{\rho \lambda}^{\rho} \Gamma_{\mu \nu}^{\lambda}-\Gamma_{\mu \lambda}^{\rho} \Gamma_{\rho v}^{\lambda}\right)$.

The variation of the action (1) with respect to the metric gives the field equations

$G_{\mu \nu}+F(\mathscr{R}) \mathscr{R}_{\mu \nu}-\frac{f(\mathscr{R})}{2} g_{\mu \nu}=\kappa^{2} T_{\mu \nu}$,

where $G_{\mu \nu}=R_{\mu \nu}-R / 2 g_{\mu \nu}$ and $R_{\mu \nu}$ are, respectively, Einstein and Ricci tensor associated with the LeviCivita connection of $g_{\mu \nu}, F(\mathscr{R}) \equiv d f / d \mathscr{R}, T_{\mu \nu}=$ $-2 / \sqrt{-g} \delta\left(\sqrt{-g} \mathscr{L}_{m}\right) / \delta g^{\mu \nu}$ is the energy-momentum tensor of the matter fields.

The variation of the action (1) with respect to the independent connection $\Gamma_{\mu \nu}^{\rho}$ yields

$\widetilde{\nabla}_{\beta}\left(\sqrt{-g} F(\mathscr{R}) g^{\mu v}\right)=0$,

where $\widetilde{\nabla}_{\beta}$ denotes the covariant derivative associated with $\Gamma_{\mu \nu}^{\rho}$. If one defines a metric $h_{\mu \nu}=F(\mathscr{R}) g_{\mu \nu}$, it can be easily shown that Eq. (4) determines a Levi-Civita connection of $h_{\mu \nu}$, which in turn can be rewritten in terms of $g_{\mu \nu}$ and its Levi-Civita connection $\left\{\begin{array}{l}\rho \\ \mu \nu\end{array}\right\}$ in the form

$\Gamma_{\mu \nu}^{\rho}=\left\{\begin{array}{l}\rho \\ \mu \nu\end{array}\right\}+\frac{1}{2}\left(\delta_{\mu}^{\rho} \partial_{\nu}+\delta_{\nu}^{\rho} \partial_{\mu}-g_{\mu \nu} g^{\rho \sigma} \partial_{\sigma}\right) \ln F(\mathscr{R})$.
Using now Eq. (5) one finds the relation between the two Ricci tensors, which is given by

$\mathscr{R}_{\mu \nu}=R_{\mu \nu}+\frac{3}{2 F^{2}} \partial_{\mu} F \partial_{\nu} F-\frac{1}{F}\left(\nabla_{\mu} \nabla_{\nu}+\frac{1}{2} g_{\mu \nu} \square\right) F$,

where $\nabla_{\mu}$ denotes the covariant derivative associated to $\{\stackrel{\rho}{\mu \nu}\}$.

Equation (6) in turn gives rise to the following relation between the two Ricci scalars:

$\mathscr{R}=R+\frac{3}{2 F^{2}}(\partial F)^{2}-\frac{3}{F} \square F$,

where $(\partial F)^{2}=g^{\alpha \beta} \partial_{\alpha} F \partial_{\beta} F$ and $\square F=g^{\alpha \beta} \nabla_{\alpha} \nabla_{\beta} F$.

The Palatini curvature $\mathscr{R}$ can be obtained from the trace of the field equation (3), which yields

$\mathscr{R} F(\mathscr{R})-2 f(\mathscr{R})=\kappa^{2} T+R \equiv X$

This trace equation can be used to express $\mathscr{R}$ algebraically in terms of $X$ when the $f(\mathscr{R})$ is given as an analytic expression. Finally, we note that the variable $X$ measures the deviation from the general relativity trace equation $R=-\kappa^{2} T$.

\section{Homogeneous Gödel-type geometries}

To make this work clear and to a certain extent self-contained, in this section we present the basic properties of homogenous Gödel-type geometries, which we use in the following sections. To this end, we first discuss the conditions for spacetime homogeneity (ST-homogeneity) of these space-times, and present all non-isometric ST-homogeneous Gödel-type classes. These ST-homogeneity conditions along with the set of isometrically non-equivalent geometries are important in the determination of ST-homogeneous Gödel-type solutions, in Sect. 4, to the hybrid metric-Palatini $f(\mathscr{R})$ field equations. Second, we discuss the existence of closed time-like curves in the metrics of these classes. The existence of these non-causal curves are crucial to examine whether hybrid metric-Palatini $f(\mathscr{R})$ gravities allow violation of causality of Gödel-type.

\subsection{Homogeneity and non-equivalent metrics}

Gödel solution to the general relativity field equations is a particular member of the broad family of geometries whose general form in cylindrical coordinates, $(r, \phi, z)$, is given by [81]

$d s^{2}=[d t+H(r) d \phi]^{2}-D^{2}(r) d \phi^{2}-d r^{2}-d z^{2}$. 
The necessary and sufficient conditions for the Gödel-type metric (9) to be space-time homogeneous (ST-homogeneous) are given by $[81,86]$

$$
\frac{H^{\prime}}{D}=2 \omega \text { and } \frac{D^{\prime \prime}}{D}=m^{2}
$$

where the prime denote derivative with respect $r$, and the parameters $(\Omega, m)$ are constants such that $\Omega^{2}>0$ and $-\infty \leq m^{2} \leq \infty$.

As a matter of fact, except for the case $m^{2}=4 \omega^{2}$ all locally ST-homogeneous Gödel-type space-times admit a group $G_{5}$ of isometries acting transitively on the whole spacetime [86]. The special case $m^{2}=4 \omega^{2}$ admits a $G_{7}$ of isometries $[86,87]$.

The irreducible set of isometrically nonequivalent SThomogeneous Gödel-type metrics can be obtained by integrating Eq. (10) and suitably eliminating nonessential integration constants. The final result is that ST-homogeneous Gödel-type geometries can be grouped in the following three classes [81]:

(i) Hyperbolic, in which $m^{2}=$ const $>0$ and

$$
H=\frac{4 \omega}{m^{2}} \sinh ^{2}\left(\frac{m r}{2}\right), \quad D=\frac{1}{m} \sinh (m r) ;
$$

(ii) Linear, in which $m=0$ and

$$
H=\omega r^{2}, \quad D=r
$$

(iii) Trigonometric, where $m^{2}=$ const $\equiv-\mu^{2}<0$ and

$$
H=\frac{4 \omega}{\mu^{2}} \sin ^{2}\left(\frac{\mu r}{2}\right), \quad D=\frac{1}{\mu} \sin (\mu r)
$$

Thus, clearly all ST-homogeneous Gödel-type geometries are characterized by the two independent parameters $m^{2}$ and $\omega$ - identical pairs $\left(m^{2}, \omega^{2}\right)$ specify isometric spacetimes [81, 86,87 . $^{2}$ In this way, to determine whether hybrid metricPalatini $f(\mathscr{R})$ gravity allows Gödel-type solutions is to find out whether its field equations can be used to specify a pair of parameters $m^{2}$ and $\omega$ for a suitably chosen matter source.

\subsection{Closed time-like curves}

We begin by noting that the presence of a single closed timelike curve in a space-time is an unequivocal manifestation of violation of causality. However, a space-time may admit

${ }^{2}$ Gödel geometry is a solution of Eintein's equations in general relativity, is indeed a particular case of the hyperbolic class of geometries in which $m^{2}=2 \omega^{2}$. non-causal closed curves other than Gödel's circles we discuss in this Section. To examine the existence of closed timelike curves in ST-homogeneous Gödel-type metrics we first rewrite the line element (9) as

$d s^{2}=d t^{2}+2 H(r) d t d \phi-d r^{2}-G(r) d \phi^{2}-d z^{2}$,

where $G(r)=D^{2}-H^{2}$. In this form it is easy to show that existence of closed time-like curves, which allows for violation of causality in homogeneous Gödel-type space-times, depends on the sign of the metric function $G(r)$. Indeed, from Eq. (14) one has that the circles, hereafter called Gödel's circles, defined by $t, z, r=$ const become closed timelike curves whenever $G(r)<0$.

For the hyperbolic $\left(m^{2}>0\right)$ class of homogeneous Gödeltype metrics, from Eqs. (11) one has that

$G(r)=\frac{4}{m^{2}} \sinh ^{2}\left(\frac{m r}{2}\right)\left[\left(1-\frac{4 \omega^{2}}{m^{2}}\right) \sinh ^{2}\left(\frac{m r}{2}\right)+1\right]$.

Therefore for $0<m^{2}<4 \omega^{2}$ there is a critical radius $r_{c}$ defined by $G(r)=0$, which is given by

$\sinh ^{2} \frac{m r_{c}}{2}=\left[\frac{4 \omega^{2}}{m^{2}}-1\right]^{-1}$,

such that for $r<r_{c}$ one has $G(r)>0$, and for $r>r_{c}$ one has $G(r)<0$. Thus, the circles $t, r, z=$ const with $r>r_{c}$ are closed timelike curves. ${ }^{3}$

For linear class $(m=0)$ of homogeneous Gödel-type space-times, from Eq. (12) one easily finds

$G(r)=r^{2}-r^{4} \omega^{2}=-r^{2}(r \omega-1)(r \omega+1)$.

Thus, there is a critical radius, defined by $G(r)=0$, and given by $r_{c}=1 / \omega$, such that for any radius $r>r_{c}$ one has $G(r)<0$, and then the circles defined by $t, z, r=$ const are closed timelike curves.

Finally for the trigonometric class $\left(m^{2}=\right.$ const $\equiv-\mu^{2}<$ 0 ), from the metric functions given by Eq. (13) one finds

$G(r)=\frac{4}{\mu^{4}} \sin ^{2}\left(\frac{\mu r}{2}\right)\left[\mu^{2}-\left(4 \omega^{2}+\mu^{2}\right) \sin ^{2}\left(\frac{\mu r}{2}\right)\right]$,

and therefore $G(r)$ has an infinite sequence of zeros. Thus, in the section $t, z, r=$ const, there is an sequence of alternating

\footnotetext{
3 The only ST-homogeneous Gödel-type space-time without these noncausal circles come about when $m^{2}=4 \omega^{2}$ (see Ref. [81]). In this case, the critical radius $r_{c} \rightarrow \infty$, and hence the violation of causality of Gödel type is avoided.
} 
causal [ $G(r)>0$ ] and noncausal $[G(r)<0$ ] regions without and with noncausal circles, depending on the value of $r=$ const (For more details see the Appendix of Ref. [75]). In this way, if $G(r)<0$ for a certain range of $r\left(r_{1}<r<r_{2}\right.$, say) noncausal Gödel's circles exist, whereas for $r$ in the next circular band $r_{2}<r<r_{3}$ (say) for which $G(r)>0$ no such closed timelike circles exist, and so on.

To close this section, we note that in this paper by noncausal and causal solutions we mean, respectively, solutions with and without violation of causality of Gödel-type, i.e., with and without Gödel's circles.

\section{Solutions in hybrid metric-Palatini gravity}

The aim of this section is twofold. First, we examine the problem of finding out ST-homogeneous solutions in hybrid metric-Palatini $f(\mathscr{R})$ gravity whose trace $T$ of the energymomentum tensor of the matter source is constant. We show that in such cases the problem of finding out solutions in the hybrid metric-Palatini $f(\mathscr{R})$ gravity reduces to the problem of determining ST-homogeneous solutions of Einstein's field equations with a cosmological constant determined by $f(\mathscr{R})$ and its first derivative $F=f^{\prime}(\mathscr{R})$. Second, we examine whether hybrid metric-Palatini $f(\mathscr{R})$ field equations admit ST-homogeneous Gödel-type solutions for a combination of a scalar field with an electromagnetic field plus a perfect fluid.

\subsection{Field equations}

We begin by noting that using Eq. (6) the field equations (3) of the hybrid metric-Palatini $f(\mathscr{R})$ gravity can be rewritten in the form

$$
G_{\mu \nu}=\kappa^{2} T_{\mu \nu}^{\mathrm{eff}}=\kappa^{2}\left(T_{\mu \nu}+T_{\mu \nu}^{\mathscr{R}}\right)
$$

where

$$
\begin{aligned}
\kappa^{2} T_{\mu \nu}^{\mathscr{R}}= & \frac{1}{2}[f(\mathscr{R})+\square F(\mathscr{R})] g_{\mu \nu}-F(\mathscr{R}) R_{\mu \nu} \\
& +\nabla_{\mu} \nabla_{\nu} F(\mathscr{R})-\frac{3}{2 F(\mathscr{R})} \partial_{\mu} F(\mathscr{R}) \partial_{\nu} F(\mathscr{R}) .
\end{aligned}
$$

In this context, an important constraint comes from the trace of the field equations (19), which can be written in the form

$$
R+\kappa^{2} T=X=-\kappa^{2} T^{\mathscr{R}}
$$

where from Eq. (20) one has

$$
\kappa^{2} T^{\mathscr{R}}=2 f(\mathscr{R})-R F(\mathscr{R})+3 \square F(\mathscr{R})-\frac{3}{2} \frac{[\partial F(\mathscr{R})]^{2}}{F(\mathscr{R})} .
$$

For ST-homogeneous spacetimes, which we are concerned with in this paper, one has that the Ricci scalar is necessarily constant. On the other hand, for matter sources whose trace of the energy-momentum $T$ is also constant, which we focus in this paper, one has $X=$ const $=-\kappa^{2} T^{\mathscr{R}}$. In such cases, one can show that the field equations of the hybrid metricPalatini gravity reduces to Einstein's field equations with a cosmological constant.

To this end, we first examine the second term on the right hand side of equation (5) which gives the departures of the independent connection $\Gamma_{\mu \nu}^{\rho}$ from Levi-Civita connection $\left\{\begin{array}{l}\rho \\ \mu \nu\end{array}\right\}$. Clearly, each individual part of this second term in this equation is proportional to

$\partial_{\mu} \ln F(\mathscr{R})=\frac{F^{\prime}}{F} \partial_{\mu} \mathscr{R}=\frac{1}{F} \partial_{\mu} F(\mathscr{R})$.

On the other hand, to calculate $\partial_{\mu} F(\mathscr{R})$ we note that from the trace equation (8) one has $\partial_{\mu} \mathscr{R}=\partial_{\mu} X /\left[\mathscr{R} F^{\prime}(\mathscr{R})-F(\mathscr{R})\right]$, which together with Eq. (23) furnishes

$\partial_{\mu} F(\mathscr{R})=\frac{F^{\prime}(\mathscr{R}) \partial_{\mu} X}{\mathscr{R} F^{\prime}(\mathscr{R})-F(\mathscr{R})}$,

provided that $\mathscr{R} F^{\prime}(\mathscr{R})-F(\mathscr{R}) \neq 0$. From Eqs. (23) and (24) one has that for $X=$ const the connection $\Gamma_{\mu \nu}^{\rho}$ reduces to Levi-Civita connection $\left\{\rho_{\mu \nu}\right\}$. Furthermore, from Eqs. (6) and (7) one can easily show that $\partial_{\mu} F(\mathscr{R})=0$ also ensures that $\mathscr{R}_{\mu \nu}=R_{\mu \nu}$ and $\mathscr{R}=R$. This makes apparent that ST-homogeneous spacetimes solutions whose trace of the energy-momentum tensor is constant $\left(R+\kappa^{2} T=X=\right.$ const) the field equations of the hybrid metric-Palatini gravity (3) reduce formally to field equations of $f(R)$ theories in the metric formalism, which can clearly be rewritten in the form

$[1+F(R)] G_{\mu \nu}-\frac{1}{2}[f(R)-R F(R)] g_{\mu \nu}=\kappa^{2} T_{\mu \nu}$,

with associated trace equation

$R F(R)-2 f(R)=\kappa^{2} T+R=$ const.

However, for an explicitly given $f(\mathscr{R})$, solving the algebraic Eq. (26) one finds constant roots $R$ 's. Thus, for each explicit root the field Eqs. (25) can be rewritten in the form ${ }^{4}$

$G_{\mu \nu}=\bar{\kappa}^{2} T_{\mu \nu}+\Lambda g_{\mu \nu}$,

\footnotetext{
${ }^{4}$ Clearly, different roots $R$ give rise to different rescales of $\kappa^{2}$, and different effective cosmological constant $\Lambda$.
} 
where

$\Lambda=\frac{f(R)-R F(R)}{2[1+F(R)]} \quad$ and $\quad \bar{\kappa}^{2}=\frac{\kappa^{2}}{1+F(R)}$.

The trace equation becomes

$R+\bar{\kappa}^{2} T+4 \Lambda=0$.

Clearly, the factor $[1+F(R)]$ in Eq. (28) is a constant that simply rescales the units of $\kappa^{2}$ and the effective cosmological constant $\Lambda$.

\subsection{Gödel-type solutions}

In this section we discuss ST-homogeneous Gödel-type solutions in hybrid metric-Palatini $f(\mathscr{R})$ gravity for wellmotivated matter contents whose trace of the energy-momentum tensor is constant.

We begin by noting that the search for ST-homogeneous Gödel-type solutions to the hybrid metric-Palatini gravity field equations is greatly simplified if instead of using coordinates basis one uses a new basis given by the following set of linearly independent one-forms (tetrad frame) $\Theta^{A}$ :

$\theta^{0}=d t+H(r) d \phi, \quad \theta^{1}=d r, \quad \theta^{2}=D(r) d \phi, \quad \theta^{3}=d z$,

relative to which the Gödel-type line element (9) takes the local Lorentzian form

$d s^{2}=\eta_{A B} \theta^{A} \theta^{B}=\left(\theta^{0}\right)^{2}-\left(\theta^{1}\right)^{2}-\left(\theta^{2}\right)^{2}-\left(\theta^{3}\right)^{2}$.

Here and in what follows capital letters are tetrad indices (or Lorentz frame indices) and run from 0 to 3 . These Lorentz frame indices are raised and lowered with Lorentz matrices $\eta^{A B}=\eta_{A B}=\operatorname{diag}(+1,-1,-1,-1)$, respectively.

In the tetrad frame (30) the nonvanishing components of the Ricci tensor, $R_{A B}=\eta^{C D} R_{C A D B}$, are given by

$R_{02}=\frac{1}{2}\left(\frac{H^{\prime}}{D}\right)^{\prime}, \quad R_{00}=\frac{1}{2}\left(\frac{H^{\prime}}{D}\right)^{2}$,

$R_{11}=R_{22}=\frac{1}{2}\left(\frac{H^{\prime}}{D}\right)^{2}-\frac{D^{\prime \prime}}{D}$,

where the prime denotes derivative with respect to $r$. Since the Lorentz frame components of the Ricci tensor depend only on $H^{\prime} / D$ and $D^{\prime \prime} / D$, from the ST-homogeneity conditions (10) one has that for all classes of ST-homogeneous Gödel-type metrics the frame components of the Ricci tensor are constants. Thus, the Ricci scalar is also constant and given by $R=\eta^{C D} R_{C D}=2\left(m^{2}-\omega^{2}\right)$.
In this Lorentzian basis the field Eq. (27) reduce to

$G_{A B}=\bar{\kappa}^{2} T_{A B}+\Lambda \eta_{A B}$,

where from Eqs. (32) and (33) along with the conditions (10) one has that the only nonvanishing Lorentz frame components of the Einstein tensor $G_{A B}$ for ST-homogeneous Gödeltype metrics take the very simple form

$G_{00}=3 \omega^{2}-m^{2}, \quad G_{11}=G_{22}=\omega^{2}, \quad G_{33}=m^{2}-\omega^{2}$.

\subsubsection{Combined-fields general solution}

In this section we take combination of scalar and electromagnetic fields with a perfect fluid as a matter source, and find a general ST-homogeneous Gödel-type solution, which contains the a perfect fluid and a scalar field particular solutions, and whose essential parameter $m^{2}$ can be $m^{2}>0$ (hyperbolic family), $m=0$ (linear class) or $m^{2}<0$ (trigonometric family) depending on the amplitude values of the matter components.

In the Lorentzian basis (30) the energy-momentum tensor of combined matter sources takes the form

$T_{A B}=T_{A B}^{(M)}+T_{A B}^{(S)}+T_{A B}^{(E M)}$,

where $T_{A B}^{(M)}, T_{A B}^{(S)}$ and $T_{A B}^{(E M)}$ are, respectively, the energy momentum tensors of a perfect fluid, a scalar field, and an electromagnetic field, which we discuss in what follows.

For a perfect fluid of of density $\rho$ and pressure $p, T_{A B}^{(M)}$ one has

$T_{A B}^{(M)}=(\rho+p) u_{A} u_{B}-p \eta_{A B}$.

The energy-momentum tensor of a single scalar field is given by

$T_{A B}^{(S)}=\Phi_{\mid A} \Phi_{\mid B}-\frac{1}{2} \eta_{A B} \Phi_{\mid M} \Phi_{\mid N} \eta^{M N}$,

where vertical bar denotes components of covariant derivatives relative to the local basis $\theta^{A}=e_{\alpha}^{A} d x^{\alpha}$ [see Eqs. (30) and (31)], i.e. $\Phi_{\mid A}=e_{\mu}^{A} \nabla_{\mu} \Phi$. Following Ref. [81] it is straightforward to show that a scalar field of the form $\Phi(z)=\varepsilon z+\epsilon$, with $\varepsilon, \epsilon=$ const, fulfills the scalar field equation $\square \Phi=\eta^{A B} \nabla_{A} \nabla_{B} \Phi=0$. Thus, the nonvanishing components of the energy-moment tensor for this scalar field are

$T_{00}^{(S)}=-T_{11}^{(S)}=-T_{22}^{(S)}=T_{33}^{(S)}=\frac{\varepsilon^{2}}{2}$,

As for the electromagnetic part of energy momentum tensor (36), following Ref. [81], the electromagnetic field tensor $F_{A B}$ given by 
$F_{03}=-F_{30}=E_{0} \sin \left[2 \omega\left(z-z_{0}\right)\right]$,

$F_{12}=-F_{21}=E_{0} \cos \left[2 \omega\left(z-z_{0}\right)\right]$,

satisfies the source-free Maxwell equations which, in the tetrad frame (30), take the form

$$
\begin{aligned}
& F_{\mid B}^{A B}+\gamma_{M B}^{A} F^{M B}+\gamma_{M C}^{C} F^{A M}=0, \\
& F_{[A B \mid C]}+2 F_{M[C} \gamma_{A B]}^{M}=0,
\end{aligned}
$$

where the brackets denote total anti-symmetrization and the Ricci rotation coefficients are defined by $\gamma_{B C}^{A}=$ $-\nabla_{\beta} \theta_{\alpha}^{A} \theta_{B}^{\alpha} \theta_{C}^{\beta}$. The non-vanishing components of the associated energy-momentum tensor $T_{A B}^{(E M)}=F_{A}^{C} F_{B C}+$ $\frac{1}{4} \eta_{A B} F^{C D} F_{C D}$ are given by

$T_{00}^{(E M)}=T_{11}^{(E M)}=T_{22}^{(E M)}=-T_{33}^{(E M)}=\frac{E_{0}^{2}}{2}$.

Thus, taking into account Eqs. (35), (37), (39) and (44), one has that for the combined-fields matter source (36) the field Eq. (34) reduce to

$$
\begin{aligned}
3 \omega^{2}-m^{2} & =\bar{\kappa}^{2}\left(\rho+\frac{\varepsilon^{2}}{2}+\frac{E_{0}^{2}}{2}\right)+\Lambda, \\
\omega^{2} & =\bar{\kappa}^{2}\left(p-\frac{\varepsilon^{2}}{2}+\frac{E_{0}^{2}}{2}\right)-\Lambda, \\
m^{2}-\omega^{2} & =\bar{\kappa}^{2}\left(p+\frac{\varepsilon^{2}}{2}-\frac{E_{0}^{2}}{2}\right)-\Lambda .
\end{aligned}
$$

To determine the essential parameters $\omega^{2}$ and $m^{2}$, we first substitute (47) into (45) to obtain

$2 \omega^{2}=\bar{\kappa}^{2}\left(\rho+p+\varepsilon^{2}\right)$

Second, we use (46), (47) and (48) to find

$m^{2}=\bar{\kappa}^{2}\left(\rho+p+2 \varepsilon^{2}-E_{0}^{2}\right)$.

The trace equation (29) gives rise to the following constrain for the cosmological constant

$2 \Lambda=\bar{\kappa}^{2}\left(p-\rho-2 \varepsilon^{2}+E_{0}^{2}\right)$

Since ST-homogeneous Gödel-type geometries are characterized by the two essential parameters $m^{2}$ and $\omega^{2}$, the above Eqs. (48) and (49) make explicit how the $f(\mathscr{R})$ gravity specifies a pair of parameters $\left(m^{2}, \omega^{2}\right)$, and therefore determines a general ST-homogeneous Gödel-type solution, for the combined-fields matter source (36).

The general solution given by Eqs. (48) and (49) contains all general relativity Gödel-type known solutions [81] as particular cases. Indeed, the perfect fluid Gödel solution is recovered when $\varepsilon=E_{0}=0$ with $\rho, p \neq 0$, whereas the scalar field causal solution [81] is retrieved for $\rho=p=E_{0}=0$ with $\varepsilon \neq 0 .^{5}$

Finally, from Eq. (49) one has that the combination of scalar plus electromagnetic field with a perfect fluid gives rise to ST-homogeneous Gödel-type solutions in hyperbolic class $\left(m^{2}>0\right)$ when $E_{0}^{2}<\rho+p+2 \varepsilon^{2}$, in the linear family $\left(m^{2}=0\right)$ for $E_{0}^{2}=\rho+p+2 \varepsilon^{2}$, and also in the trigonometric class $\left(m^{2}<0\right)$ when $E_{0}^{2}>\rho+p+2 \varepsilon^{2}$. Moreover, from Eq. (49) we also have that these three families of ST-homogeneous Gödel-type solutions can also be generated with a simple combination of scalar and electromagnetic fields (thus for $\rho=p=0$ ), depending on the relative values of the amplitudes $\varepsilon^{2}$ and $E_{0}^{2}$, which give the sign of the term $2 \varepsilon^{2}-E_{0}^{2}$ in Eq. (49).

\section{Concluding remarks}

Despite the great success of the general relativity theory, a good deal of effort has recently gone into the study of the socalled modified gravity theories. In the cosmological modeling framework, this is motivated by the fact that these theories provide an alternative way to explain the current accelerating expansion of the Universe with no need to invoke the dark energy matter component. The hybrid metric-Palatini $f(\mathscr{R})$ gravity is a recently devised approach to such modified theories, in which it is added to the ordinary Ricci scalar $R$, in the Einstein-Hilbert Lagrangian, a function, $f(\mathscr{R})$, of Palatini curvature scalar $\mathscr{R}$, which is constructed from the independent connection $\Gamma_{\mu \nu}^{\rho}$.

In general relativity, the bare existence of the Gödel solution to Einstein's equations, for a physically well-motivated perfect-fluid source, makes it apparent that this theory permits solutions with violation of causality on nonlocal scale, regardless of its local Lorentzian character that ensures the local validation of the causality principle. In the context of the hybrid metric-Palatini $f(\mathscr{R})$ gravity theories the spacetime manifolds are also assumed to be locally Lorentzian. Hence the chronology and causality structure of special relativity are inherited locally. The nonlocal question, however, is left open, and violation of causality can in principle arise.

Since homogeneous Gödel-type geometries necessarily lead to the existence of closed timelike circles (Sect. 3.2), which is an unequivocal manifestation of violation of causality, a natural way to tackle this question is by investigating whether the hybrid metric-Palatini $f(\mathscr{R})$ gravity theories permit Gödel-type solutions to their field equations. Further-

\footnotetext{
$\overline{5}$ We note that Gödel metric $\left(m^{2}=2 \omega^{2}\right)$ can also be generated through a particular combination of perfect fluid with a scalar field, namely for non-vanishing equal amplitude of the scalar and electromagnetic fields $\varepsilon=E_{0} \neq 0$. Indeed in this case Eqs. (48) and (49) give $m^{2}=2 \omega^{2}=$ $\bar{\kappa}^{2}\left(\rho+p+\varepsilon^{2}\right)$.
} 
more, if gravity is to be governed by a $f(\mathscr{R})$ there are a number of issues ought to be reexamined in its context, including the question as to whether these theories admit Gödel-type solutions, or would remedy the violation of causality problem by ruling out this type of solutions, which are permitted in general relativity.

In this article, to proceed further with the investigations on the potentialities, difficulties and limitations of $f(\mathscr{R})$, we have examined whether $f(\mathscr{R})$ gravity theories admit homogeneous Gödel-type solutions for physically well-motivated matter sources. To this end, we have first examined the problem of finding out ST-homogeneous solutions in these hybrid gravity theories whose trace $T$ (invariant) of the energymomentum tensor is constant. We have shown that under this assumption the problem of finding out solutions in the hybrid metric-Palatini $f(\mathscr{R})$ gravity reduces to the problem of determining ST-homogeneous solutions of Einstein's field equations with a cosmological constant. Employing this farreaching simplifying result, we first have found a general Gödel-type solution for a source that is a combination of scalar and electromagnetic fields with a perfect fluid. In this general Gödel-type solution solution the essential parameter $m^{2}$ can be $m^{2}>0$ (hyperbolic family), $m=0$ (linear class) or $m^{2}<0$ (trigonometric family) depending on the values of the amplitudes $\varepsilon$ (scalar field) and $E_{0}$ (electromagnetic field), and the density $\rho$ and pressure $p$ of the perfect fluid. This general homogeneous Gödel-type solution also contains previously known solutions of the literature as special cases. There emerges from one of the particular solution of the hyperbolic family that every perfect-fluid Gödel-type solution of any $f(\mathscr{R})$ gravity with density $\rho$ and pressure $p$ and satisfying the weak energy conditions $\rho>0$ and $\rho+p \geq 0$ is necessarily isometric to the Gödel geometry. This extends to the context of $f(\mathscr{R})$ gravity a theorem established in the context of the general relativity, which states that Gödel solution is the sole perfect fluid solution of Einstein's equations [85].

Whether or not the physical laws permit the existence of stable time machines in the form of closed timelike curves is a research field in general relativity and other gravity theories. Violation of causality on the other hand raises intriguing logical paradoxes, and is generally seen as undesirable feature in physics. The two most known remedies to these paradoxes are Novikov's self-consistency principle [88-90], which was designed to reconcile the logical inconsistencies by demanding that the only admissible local solutions are those which are globally self-consistent, ${ }^{6}$ and Hawking's chronology protection conjecture [91], which suggests that even though closed timelike curves are classically possible to be produced, quantum effects are likely to prevent such time

\footnotetext{
${ }^{6}$ Clearly this principle is ultimately an ad hoc global topological constraint on admissible solutions of gravity theories, thus beyond the standard scope of the local formulation of the gravity theories.
}

travel. In this way, the laws of quantum physics would prevent closed timelike curves from appearing. ${ }^{7}$ In this regard, Hawking and Penrose have also pointed out that severe causality assumptions could risk 'ruling out something that gravity is trying to tell us' [94], thus, discouraging further investigations. The possible existence of closed timelike curves is also particularly interesting in the quantum realm, where, for example, the quantum systems traversing these curves have been studied [95] and experimental simulation of closed timelike curves have been undertaken [96].

To conclude, we emphasize that the bare existence of the ST-homogeneous Gödel-type solutions that we have found makes apparent that the hybrid metric-Palatini $f(\mathscr{R})$ gravity does not remedy at a classical level the causal pathology in the form of closed timelike curves that arises in the context of general relativity. We are not aware of a quantum gravity theory following the hybrid metric-Palatini structure, though.

Acknowledgements M.J. Rebouças acknowledges the support of FAPERJ under a CNE E-26/202.864/2017 Grant, and thanks CNPq for the grant under which this work was carried out. J. Santos acknowledges support of Programa de Pós-Graduação em Física-CCET/UFRN.

Open Access This article is distributed under the terms of the Creative Commons Attribution 4.0 International License (http://creativecomm ons.org/licenses/by/4.0/), which permits unrestricted use, distribution, and reproduction in any medium, provided you give appropriate credit to the original author(s) and the source, provide a link to the Creative Commons license, and indicate if changes were made.

Funded by $\mathrm{SCOAP}^{3}$.

\section{References}

1. A.G. Riess et al., Observational evidence from supernovae for an accelerating universe and a cosmological constant. Astron. J. 116, 1009 (1998)

2. S. Perlmutter et al., Discovery of a supernova explosion at half the age of the Universe. Nature 391, 51 (1998)

3. S. Perlmutter et al., Measurements of $\Omega$ and $\Lambda$ from 42 highredshift supernovae. Astrophys. J. 517, 565 (1999)

4. R. Adam et al., Planck 2015 results. I. Overview of products and scientific results. Astron. Astrophys. 594, A1 (2016)

5. D.N. Spergel et al., Three-year Wilkinson Microwave Anisotropy Probe (WMAP) observations: implications for cosmology. Astrophys. J. Suppl. 170, 377S (2007)

6. S. Cole et al., The $2 \mathrm{dF}$ Galaxy Redshift Survey: power-spectrum analysis of the final data set and cosmological implications. Mon. Not. R. Astron. Soc. 362, 505 (2005)

7. D.J. Eisenstein et al., Detection of the Baryon acoustic peak in the large-scale correlation function of SDSS luminous red galaxies. Astrophys. J. 633, 560 (2005)

8. W.J. Percival et al., Baryon acoustic oscillations in the Sloan digital sky survey data release 7 galaxy sample. Mon. Not. R. Astron. Soc. 401, 2148 (2010)

\footnotetext{
${ }^{7}$ For a good pedagogical overview with a fair list of references on the chronology protection conjecture and Novikov's self-consistency principle see Visser [92], and the recent review article on closed timelike curves and violation of causality by Lobo [93].
} 
9. C. Blake et al., The Wiggle Z Dark Energy Survey: mapping the distance-redshift relation with baryon acoustic oscillations. Mon. Not. R. Astron. Soc. 418, 1707 (2011)

10. L. Anderson et al., The clustering of galaxies in the SDSS-III Baryon Oscillation Spectroscopic Survey: Baryon acoustic oscillations in the data release 9 spectroscopic galaxy sample. Mon. Not. R. Astron. Soc. 428, 1036 (2013)

11. A. De Felice, $S$. Tsujikawa, $\mathrm{f}(\mathrm{R})$ Theories. Living Rev. Rel. 13, 3 (2010)

12. T.P. Sotiriou, V. Faraoni, $f(R)$ theories of gravity. Rev. Mod. Phys. 82, $451(2010)$

13. S. Nojiri, S.D. Odintsov, Unified cosmic history in modified gravity: from $\mathrm{F}(\mathrm{R})$ theory to Lorentz non-invariant models. Phys. Rep. 505, 59 (2011)

14. G.J. Olmo, Palatini approach to modified gravity: $f(R)$ theories and beyond. Int. J. Mod. Phys. D 20, 413 (2011)

15. S. Capozziello, M. De Laurentis, Extended theories of gravity. Phys. Rep. 509, 167 (2011)

16. S. Capozziello, V. Faraoni, Beyond einstein gravity, fundamental theories of physics, vol 170 (Springer, Dordrecht, 2011)

17. W. Hu, I. Sawicki, Models of $f(R)$ cosmic acceleration that evade solar system tests. Phys. Rev. D 76, 064004 (2007)

18. S. Tsujikawa, Observational signatures of $f(R)$ dark energy model that satisfy cosmological and local gravity constraints. Phys. Rev. D 77, 023507 (2008)

19. T. Koivisto, H. Kurki-Suonio, Cosmological perturbations in the Palatini formulation of modified gravity. Class. Quantum Gravity 23, 2355 (2006)

20. T. Koivisto, The matter power spectrum in $f(R)$ gravity. Phys. Rev. D 73, 083517 (2006)

21. G.J. Olmo, Violation of the equivalence principle in modified theories of gravity. Phys. Rev. Lett. 98, 061101 (2007)

22. G.J. Olmo, Hydrogen atom in Palatini theories of gravity. Phys. Rev. D 77, 084021 (2008)

23. T. Harko, T.S. Koivisto, F.S.N. Lobo, G.J. Olmo, Metric-Palatini gravity unifying local constraints and late-time cosmic acceleration. Phys. Rev. D 85, 084016 (2012)

24. S. Capozziello, T. Harko, F.S.N. Lobo, G.J. Olmo, Hybrid modified gravity unifying local tests, galactic dynamics and late-time cosmic acceleration. Int. J. Mod. Phys. D 22, 1342006 (2013)

25. S. Capozziello et al., Cosmology of hybrid metric-Palatini $f(X)-$ gravity. J. Cosmol. Astropart. Phys. 04, 011 (2013)

26. S. Capozziello et al., The virial theorem and the dark matter problem in hybrid metric-Palatini gravity. J. Cosmol. Astropart. Phys. 07, 024 (2013)

27. S. Capozziello et al., Galactic rotation curves in hybrid metricPalatini gravity. Astropart. Phys. 50, 65 (2013)

28. S. Capozziello et al., Hybrid metric-Palatini gravity. Universe 1, 199 (2015)

29. N.A. Lima, V.S. Barreto, Constraints on hybrid metric-Palatini gravity from background evolution. Astrophys. J. 818, 186 (2016)

30. S. Capozziello et al., Wormholes supported by hybrid metricPalatini gravity. Phys. Rev. D 86, 127504 (2012)

31. C.G. Böhmer, F.S.N. Lobo, N. Tamanini, Einstein static universe in hybrid metric-Palatini gravity. Phys. Rev. D 88, 104019 (2013)

32. N.A. Lima, Dynamics of linear perturbations in the hybrid metricPalatini gravity. Phys. Rev. D 89, 083527 (2014)

33. S. Capozziello et al., The Cauchy problem in hybrid metric-Palatini $f(X)$-gravity. Int. J. Geom. Methods Mod. Phys. 11, 1450042 (2014)

34. S. Carloni, T. Koivisto, F.S.N. Lobo, Dynamical system analysis of hybrid metric-Palatini cosmologies. Phys. Rev. D 92, 064035 (2015)

35. Fu Qi-Ming et al., Hybrid metric-Palatini brane system. Phys. Rev. D 94, 024020 (2016)
36. A. Borowiec et al., Invariant solutions and Noether symmetries in hybrid gravity. Phys. Rev. D 91, 023517 (2015)

37. T. Azizi, N. Borhani, Thermodynamics in hybrid metric-Palatini gravity. Astrophys. Space Sci. 357, 146 (2015)

38. W.J. van Stockum, The gravitational field of a distribution of particles rotating around an axis of symmetry. Proc. R. Soc. Edinb. 57, 135 (1937)

39. M.S. Morris, K.S. Thorne, U. Yurtsever, Wormholes, time machines, and the weak energy condition. Phys. Rev. Lett. 61, 1446 (1988)

40. M.S. Morris, K.S. Thorne, Wormholes in spacetime and their use for interstellar travel: a tool for teaching general relativity. Am. J. Phys. 56(1988), 395 (1988)

41. F.J. Tipler, Rotating cylinders and the possibility of global causality violation. Phys. Rev. D 9, 2203 (1974)

42. J.R. Gott III, Closed timelike curves produced by pairs of moving cosmic strings: exact solutions. Phys. Rev. Lett. 66, 1126 (1991)

43. M. Alcubierre, The warp drive: hyper-fast travel within general relativity. Class. Quantum Gravity 11, L73 (1994)

44. K. Gödel, An example of a new type of cosmological solutions of Einstein's field equations of gravitation. Rev. Mod. Phys. 21, 447 (1949)

45. M.M. Som, A.K. Raychaudhuri, Cylindrically symmetric charged dust distributions in rigid rotation in general relativity. Proc. R. Soc. Lond. A 304, 81 (1968)

46. A.K. Raychaudhuri, S.N. Guha Thakurta, Homogeneous spacetimes of the Gödel-type. Phys. Rev. D 22, 802 (1980)

47. M.J. Rebouças, J.E. Åman, A.F.F. Teixeira, A note on Gödel-type space-times. J. Math. Phys. 27, 1370 (1986)

48. M.J. Rebouças, A.F.F. Teixeira, Features of a relativistic space-time with seven isometries. Phys. Rev. D 34, 2985 (1986)

49. F.M. Paiva, M.J. Rebouças, A.F.F. Teixeira, Time travel in the homogeneous Som-Raychaudhuri universe. Phys. Lett. A 126, 168 (1987)

50. A. Krasiński, Rotating dust solutions of Einsteins equations with 3dimensinal symmetry groups-III. All killing fields linearly independent of U(alpha) and W(alpha). J. Math. Phys. 39, 2148 (1998)

51. S. Carneiro, A Gödel-Friedman cosmology? Phys. Rev. D 61, $083506(2000)$

52. Y.N. Obukhov, On physical foundations and observational effects of cosmic rotation, in Colloquium on cosmic rotation, ed. by $\mathrm{M}$. Scherfner, T. Chrobok, M. Shefaat (Wissenschaft und Technik, Berlin, 2000), pp. 23-96. arXiv:astro-ph/0008106

53. J.D. Barrow, C.G. Tsagas, Dynamics and stability of the Gödel universe. Class. Quantum Gravity 21, 1773 (2004)

54. M.P. Dabrowski, J. Garecki, Energy momentum and angular momentum of Gödel universes. Phys. Rev. D 70, 043511 (2004)

55. L.L. Smalley, Gödel cosmology in Riemann-Cartan spacetime with spin density. Phys. Rev. D 32, 3124 (1985)

56. J. Duarte de Oliveira, A.F.F. Teixeira, J. Tiomno, Homogeneous Cosmos of Weyssenhoff fluid in Einstein-Cartan space. Phys. Rev. D 34, 3661 (1986)

57. A.J. Accioly, G.E.A. Matsas, Are there causal vacuum solutions with the symmetries of the Gödel universe in higher-derivative gravity? Phys. Rev. D 38, 1083 (1988)

58. J.D. Barrow, M.P. Dabrowski, Gödel universes in string theory. Phys. Rev. D 58, 103502 (1998)

59. J.E. Åman, J.B. Fonseca-Neto, M.A.H. MacCallum, M.J. Rebouças, Riemann-Cartan spacetimes of Gödel-type. Class. Quantum Gravity 15, 1089 (1998)

60. M.J. Rebouças, A.F.F. Teixeira, Riemannian space-times of Gödel type in five dimensions. J. Math. Phys. 39, 2180 (1998)

61. M.J. Rebouças, A.F.F. Teixeira, Causal anomalies in Kaluza-Klein gravity theories. Int. J. Mod. Phys. A 13, 3181 (1998)

62. P. Kanti, C.E. Vayonakis, Gödel-type universes in string-inspired charged gravity. Phys. Rev. D 60, 103519 (1999) 
63. H.L. Carrion, M.J. Rebouças, A.F.F. Teixeira, Gödel-type spacetimes in induced matter gravity theory. J. Math. Phys. 40, 4011 (1999)

64. E.K. Boyda, S. Ganguli, P. Horava, U. Varadarajan, Holographic protection of chronology in universes of the Gödel type. Phys. Rev. D 67, 106003 (2003)

65. J.D. Barrow, C.G. Tsagas, The Gödel brane. Phys. Rev. D 69, 064007 (2004)

66. M. Banados, G. Barnich, G. Compere, A. Gomberoff, Threedimensional origin of Gödel spacetimes and black holes. Phys. Rev. D 73, 044006 (2006)

67. W.-H. Huang, Instability of tachyon supertube in type IIA Gödel spacetime. Phys. Lett. B 615, 266 (2005)

68. D. Astefanesei, R.B. Mann, E. Radu, Nut charged space-times and closed timelike curves on the boundary. JHEP 01, 049 (2005)

69. Y. Brihaye, J. Kunz, E. Radu, From black strings to black holes: nuttier and squashed $\mathrm{AdS}_{5}$ solutions. JHEP 08, 025 (2009)

70. C. Furtado et al., Gödel solution in modified gravity. Phys. Rev. D 79, 124039 (2009)

71. M.J. Rebouças, J. Santos, Gödel-type universes in $\mathrm{f}(\mathrm{R})$ gravity. Phys. Rev. D 80, 063009 (2009)

72. J. Santos, M.J. Rebouças, T.B.R.F. Oliveira, Gödel-type universes in Palatini $\mathrm{f}(\mathrm{R})$ gravity. Phys. Rev. D 81, 123017 (2010)

73. Z. Tao, W. Pu-Xun, Yu. Hong-Wei, Gödel-type universes in $f(R)$ gravity with an arbitrary coupling between matter and geometry. Chin. Phys. Lett. 28, 120401 (2011)

74. D. Liu, P. Wu, H. Yu, Gödel-type universes in $\mathrm{f}(\mathrm{T})$ gravity. Int. J. Mod. Phys. D 21, 1250074 (2012)

75. J.B. Fonseca-Neto, AYu. Petrov, M.J. Rebouças, Gödel-type universes and chronology protection in Hořava-Lifshitz gravity. Phys. Lett. B 725, 412 (2013)

76. P.J. Porfírio et al., Chern-Simons modified gravity and closed timelike curves. Phys. Rev. D 94, 044044 (2016)

77. J.A. Agudelo et al., Gödel and Gödel-type universes in BransDicke theory. Phys. Lett. B 762, 96 (2016)

78. M. Gürses, Ç. Şentürk, Gödel-type metrics in Einstein-Aether theory II: nonflat background in arbitrary dimensions. Gen. Relativ. Grav. 48, 63 (2016)

79. Otalora, M.J. Rebouças, Violation of causality in $f(T)$ gravity. Eur. Phys. J. C 77, 799 (2017)

80. T. Clifton, J.D. Barrow, The existence of Gödel, Einstein, and de Sitter universes. Phys. Rev. D 72, 123003 (2005)
81. M.J. Rebouças, J. Tiomno, Homogeneity of Riemannian spacetimes of Gödel type. Phys. Rev. D 28, 1251 (1983)

82. B.P. Abbott et al., Virgo, LIGO scientific. Phys. Rev. Lett. 116, 061102 (2016)

83. B.P. Abbott et al., Virgo, LIGO scientific. Phys. Rev. Lett. 119, 161101 (2017)

84. B.P. Abbott et al., Virgo, Fermi-GBM, integral. Astrophys. J. 848, L13 (2017)

85. F. Bampi, C. Zordan, A note on Gödel's metric. Gen. Relativ. Grav. 9, 393 (1978)

86. M.J. Rebouças, J.E. Åman, Computer-aided study of a class of Riemannian space-times. J. Math. Phys. 28, 888 (1987)

87. A.F.F. Teixeira, M.J. Rebouças, J.E. Åman, Isometries of homogeneous Gödel-type spacetimes. Phys. Rev. D 32, 3309 (1985)

88. I.D. Novikov, Time machine and selfconsistent evolution in problems with selfinteraction. Phys. Rev. D 45, 1989 (1992)

89. A. Carlini, V.P. Frolov, M.B. Mensky, I.D. Novikov, H.H. Soleng, Time machines: the principle of selfconsistency as a consequence of the principle of minimal action. Int. J. Mod. Phys. D 4, 557 (1995)

90. A. Carlini, I.D. Novikov, Time machines and the principle of selfconsistency as a consequence of the principle of stationary action. II: the Cauchy problem for a self-interacting relativistic particle. Int. J. Mod. Phys. D 5, 445 (1996)

91. S.W. Hawking, Chronology protection conjecture. Phys. Rev. D 46, 603 (1992)

92. M. Visser, The quantum physics of chronology protection, in The future of theoretical physics and cosmology: celebrating Stephen Hawking's 60th birthday, ed. by G.W. Gibbons, E.P.S. Shellard, S.J. Rankin (Cambridge U.P, Cambridge, 2003), pp. 161-175

93. F.S. Lobo, Closed timelike curves and causality violation, classical and quantum gravity: theory, analysis and applications (Nova Science Publisher, Hauppauge, 2008)

94. S. Hawking, R. Penrose, The nature of space and time (Princeton University Press, Princeton, 1996)

95. D. Deutsch, Quantum mechanics near closed timelike lines. Phys. Rev. D 44, 3197 (1991)

96. M. Ringbauer et al., Experimental simulation of closed timelike curves. Nat. Commun. 5, 4145 (2014) 